\title{
Editorial
}

\section{Foreword to special section on VRIPHYS 2015}

The workshop on Virtual Reality Interactions and Physical Simulations (VRIPHYS) is one of the well-established international conferences in computer animation and virtual reality. Its goal is to attract high-quality research papers in the domains of physical simulation and dynamic interaction in virtual reality environments. We also welcome papers showing on-going research with promising results and new technology with applications of related focus.

Since 2004, this annual workshop has provided an opportunity for researchers in computer animation and virtual reality to present and discuss their latest results, and to share ideas for potential directions of future research. The first workshop was held in Colima (Mexico), followed by successful venues in Pisa (Italy), Madrid (Spain), Dublin (Ireland), Grenoble (France), Karlsruhe (Germany), Copenhagen (Denmark), Lyon (France), Darmstadt (Germany), Lille (France) and Bremen (Germany).

In 2015, the 12th VRIPHYS was back to Lyon, hosted by the University of Lyon 1 and the LIRIS research laboratory, in cooperation with Eurographics (EG). The workshop featured a technical program including 15 accepted full papers, posters, or short presentations of work in progress. Each paper has been evaluated by a double-blind process by at least three expert reviewers from the field.

This year, two high-level keynote presentations enlightened the event, one by Arjan EGGES (Universiteit Utrecht, Virtual Human Technology Lab, Netherlands) and the other by Jean-Rémy CHARDONNET (Arts et Métiers ParisTech, Institut Image, France). We were pleased and grateful that they accepted our invitation. The program was completed by demonstrations of industrial solutions and open-source software.

Among the finally accepted manuscripts, only the three best papers made it to this special section. They have been selected based on the reviews, the presentation, and their prospective impact on academia and industry. After the conference, its authors were invited to submit an extended and augmented version of their conference paper to $\mathrm{C} \& \mathrm{G}$. For these submissions, five reviewers scrutinized each revised paper both for technical content and, in particular, the amount of additional material submitted.

The first manuscript in this special issue, Accurate and adaptive contact modeling for multi-rate multi-point haptic rendering of static and deformable environments, by Knott et al. [1], is the winner of the best paper award of the conference. It tackles the notorious difficult problem of haptic rendering of multiple unilateral contact constraints. The contributions focus on two aspects of the algorithm: (i) contact determination in the context of constraint-based contact handling, and (ii) intermediate representations for multi-rate haptic rendering. This is a promising method in between collision detection and contact solving. The second paper, GPU accelerated grid-free surface tracking, by
Chentanez et al. [2] presents a parallel implementation of a meshbased surface tracker running on the GPU using recent features like dynamic parallelism. The algorithm is guaranteed to produce a manifold mesh without boundary, and the fact that it is fast and completely grid-free makes it suitable for the use in large, unbounded domains. The third paper by Brousset et al. focuses on Simulation and control of breaking waves using an external force [3]. It allows both for breaking waves and crossing of multiple waves. The paper proposes supplementary tools to control wave generation that enable digital artists to create complex and more realistic phenomena in a simple manner.

We are grateful to Prof. J. Jorge and all of the C\&G journal staff for facilitating the publication of this special section. We would like to thank the authors and the reviewers that greatly helped us with the revision of the manuscripts, for contributing to the success of the special issue. The organizing committee would like to acknowledge the continuous and great help of S. Behnke of EuroGraphics for making this possible. We also thank NVIDIA for sponsoring the Best Paper Award with a high-end Quadro M6000 graphics card, and a special thanks to J. Bender for its valuable help and advice. For further information about VRIPHYS 2015, please visit the official website http://vriphys2015.sciencesconf.org/.

\section{References}

[1] Knott TC, Kuhlen TW. Accurate and adaptive contact modeling for multi-rate multi-point haptic rendering of static and deformable environments. Comput Graph 2016;57:68-80.

[2] Chentanez N, Müller M, Macklin M. GPU accelerated grid-free surface tracking. Comput Graph 2016;57:1-11.

[3] Brousset M, Darles E, IMeneveaux D, Poulin P, Crespin B. Simulation and control of breaking waves using an external force. Comput Graph 2016;57: 102-11.

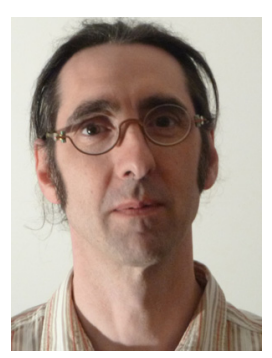

Fabrice Jaillet is an associate professor since 1999 at LIRIS Lab, Université Lyon1, France. He graduated as engineer from INSA-Lyon in Computer Science in 1994, and defended his Ph.D. in 1998 in mesh reconstruction from medical imaging for simulating deformable objects. He has been formerly head of the Computer Science department, IUT Lyon1, and is now deputyhead of the SAARA research team. He also spent three years in Chile, at the CMM in Santiago and DIM in Concepción, on developing new numerical methods for simulating soft tissues for medical applications. His research includes computational geometry, animation for computer graphics, topological and physicallybased modeling of 3D deformable objects, biomechanical simulation of soft tissues, parallel algorithms, augmented reality, haptic devices. He has strong expertise 
in simulation for medical applications, in particular for computer assisted treatment planning and operation, and training simulators.

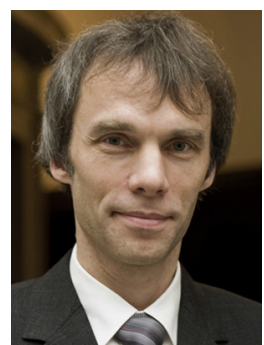

Gabriel Zachmann is a professor for computer graphics, visual computing, and virtual reality at University of Bremen, Germany, since 2012. Before that, he established and headed the computer graphics group at Clausthal University, Germany, where he was a professor with the computer science department since 2005. His research interests include geometric algorithms for computer graphics, in particular collision detection and proximity computations, massivelyparallel algorithms on the GPU, virtual prototyping, in particular virtual assembly simulation, virtual reality in general, computer vision based hand tracking, algorithms in haptics and force-feedback, immersive user interaction, immersive visualization, virtual cities, and others.

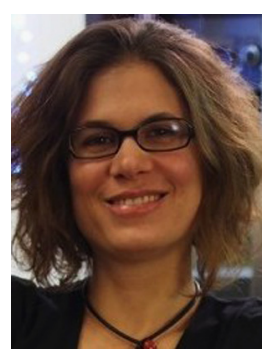

Florence Zara is an associate professor since 2005 for computer graphics, animation and virtual reality at LIRIS, Université Lyon 1, France. Before that, she defended her Ph.D. in Computer Sciences at Grenoble in 2003. It combined high performance computing, physical modeling and Virtual Reality in the ID-IMAG laboratory. Then, she spent two years at LSIIT, Strasbourg, in 4D data visualization. Her research interests include animation for computer graphics, topological and physical modeling of 3D deformable objects, parallel algorithms, biomechanical simulation of soft tissues, mechanical simulation of pregnant pelvic system, the coupling of numerical simulations and haptic devices, the realization of training simulators for medical gestures, and others.
Fabrice Jaillet*, Florence Zara

LIRIS, Université Lyon 1, France

E-mail addresses: fabrice.jaillet@liris.cnrs.fr, florence.zara@liris.cnrs.fr

Gabriel Zachmann

University of Bremen, Germany E-mail address: zach@cs.uni-bremen.de

* Corresponding author. 